\title{
El diseño de ofertas de información basado en la experiencia de usuarios. El caso del boletín Reportes de Información.
}

\section{The design of information offers based on user experience. The case of Reportes de Información bulletin.}

\section{Luis Ernesto Paz Enrique ${ }^{1}$, Eduardo Alejandro Hernández Alfonso², Deymis Tamayo Rueda ${ }^{3}$, Maylín Frías Guzmán ${ }^{4}$.}

\section{Resumen}

En el estudio se analizan aspectos que deben considerarse para el diseño de ofertas de información como la experiencia de los usuarios, sus características y necesidades. La filial de la Asociación Cubana de Bibliotecarios en Villa Clara, Cuba; carece de un órgano oficial de divulgación para sus asociados. Se plantean como objetivos del estudio identificar las características de los asociados de la organización y diseñar una oferta de información Web para la filial de la Asociación Cubana de Bibliotecarios en Villa Clara a partir de las características y la experiencia de los usuarios. Se emplean métodos en los niveles teórico y empírico para la recogida de información. Se realiza un estudio de usuarios que contempla aspectos para facilitar la identificación de las fuentes que más se emplean para la publicación de artículos, la consulta de información, la investigación científica y las temáticas de investigación fundamentales. Se diseña una oferta de información basada en la experiencia y características de los usuarios.

\section{Palabras clave}

Diseño de ofertas de información, estudio de usuarios, experiencia de usuario, Reportes de Información, Asociación Cubana de Bibliotecarios.

\footnotetext{
${ }^{1}$ Licenciado en Ciencias de la Información. Especialista en Gestión Documental. Dirección de Recursos Humanos. Universidad Central "Marta Abreu” de Las Villas. E-mail: luisernestope@uclv.cu

2 Licenciado en Comunicación Social. Sala de Historia. Departamento de Extensión Universitaria. Universidad Central "Marta Abreu” de Las Villas. E-mail: ealejandro@uclv.cu

${ }^{3}$ Licenciada en Ciencias de la Información. Profesora del Departamento de Ciencias de la Información. Facultad de Matemática, Física y Computación. Universidad Central "Marta Abreu” de Las Villas. E-mail: deymist@uclv.cu

${ }^{4}$ Máster en Ciencias de la Información. Jefa de la Carrera de Ciencias de la Información. Departamento de Ciencias de la Información. Facultad de Matemática, Física y Computación. Universidad Central "Marta Abreu" de Las Villas. E-mail: maylinfo@uclv.cu
} 


\begin{abstract}
The study analyzed aspects to consider for the design of information offers based on user experience, needs and characteristics. The affiliate of the Cuban Association of Librarians in Villa Clara, Cuba; does not have an official organ of disclosure for its members. It is presented as objectives of the study to identify the characteristics the associated of the organization and design an information offer to the affiliate of the Cuban Association of Librarians in Villa Clara from the characteristics and user experience. Methods are used in the theoretical and empirical levels for the collection of information. Is realized a user study which includes aspects to facilitate identification of the sources most frequently used for the publication of articles, information consulting, scientific research and the main themes of research. Is designed an information offer of based on experience and user characteristics.
\end{abstract}

\title{
Keywords
}

Information offers design, User study, User experience, Information Reports, Cuban Association of Librarians.

Recibido: 16/11/2015

Aceptado: $12 / 03 / 2016$

DOI: http:/ /dx.doi.org/10.5557/IIMEI7-N12-089116

Descripción propuesta: PAZ ENRIQUE, Luis Ernesto; HERNÁNDEZ ALFONSO, Eduardo Alejandro; TAMAYO RUEDA, Deymis; FRÍAS GUZMÁN, Maylín, 2016. El diseño de ofertas de información basado en la experiencia de usuarios. El caso del boletín Reportes de Información. Métodos de Información, 7(12), 89-116.

\section{Introducción}

El vertiginoso desarrollo de las Tecnologías de la Información y las Comunicaciones (TIC) han propiciado un cambio en los formatos y modos en los que los usuarios buscan, recuperan y usan la información. El profesional de la información debe, ante el fenómeno tecnológico, adaptarse a los cambios para ofrecer productos y servicios que satisfagan las necesidades de los usuarios. El investigador Calva-González (2013) plantea que los usuarios son la razón por la cual la información debe conservarse, 
para empleo además de las siguientes generaciones. Además para promover el avance en las ramas del saber y en la sociedad misma.

El profesional de la información debe superarse para el diseño de servicios y productos, debido a las nuevas tipologías de usuarios que existen actualmente. Uno de los mayores retos por los que atraviesa la sociedad de la información son las brechas digitales. Las brechas digitales se identifican por el papel que juega el usuario ante las TIC.

El diseño de servicios y productos (binomio declarado en lo sucesivo como ofertas de información) deben generarse en la identificación de los usuarios, el estudio de sus características y la construcción colectiva. Producto de las TIC, las ofertas de información emplean recursos electrónicos para satisfacer las demandas de los usuarios. Así mismo, el auge de la Web 2.0 y su filosofía de elaboración colectiva y socialización por parte de los usuarios de la Web, son enfoques presentes en la actualidad cuando se diseñan ofertas de información.

El investigador Rey-Martín (2000) plantea que "es mejor emplear como indicador de satisfacción de usuarios, la utilización efectiva de los servicios, con lo que se otorga a los usuarios un papel activo en el proceso de transferencia de la información al considerarlo como un coproductor del servicio y de la calidad del mismo".

Para el diseño de ofertas de información es necesario el uso de principios de diversas disciplinas como la Arquitectura de Información (AI), encargada de organizar y estructurar la información en espacios digitales. La visualidad es una de las características fundamentales para los resultados de la AI. El diseño de interfaces debe estar acompañado de elementos visuales que le resulten familiares a los usuarios. Para esto el diseño gráfico se encarga de los elementos visuales que debe acompañar la $\mathrm{AI}$ de las ofertas.

Toda oferta que se diseñe debe partir de las necesidades de información de sus usuarios. Un diseño basado en la experiencia de los usuarios garantizará la usabilidad y demandará menor cantidad de recursos destinados al entrenamiento. Sobre las necesidades de información y las TIC para el diseño de servicios se identifican varios autores como Gallo (2008), Rendón-Rojas (2010), Belimpasakis y Saaranen (2010), Figueredo et al (2013), Harsh y Mishra (2012), Wang y Klinc (2012), Cabrera et al (2010), González, (2011). La aplicación de un servicio de divulgación- información es la vía o canal para 
que los usuarios puedan recibir la información que necesitan y así satisfacer sus demandas. Una oferta centrada en el usuario y que utilice las bondades tecnológicas, propiciará el intercambio, la colaboración (Manso-Rodríguez, 2012) y el establecimiento de relaciones sociales.

La filial de la Asociación Cubana de Bibliotecarios (ASCUBI) en Villa Clara carece de un órgano oficial de divulgación para sus asociados. Se registran después de la creación de esta filial, tres boletines que circularon por un período efímero. Desde 1992 la filial no posee un órgano de divulgación oficial, aunque circularon otras publicaciones seriadas que de cierta manera reflejaron el acontecer de la organización. Por estas razones, el deseo de crear un boletín para esta asociación fue manifestado por varios de los asociados que en muchos casos tomaron iniciativa propia para crear un boletín. La ausencia de un órgano de divulgación oficial de la filial constituye una carencia para la divulgación de sus informaciones culturales, promover la investigación científica y la superación de sus asociados. Se plantean como objetivos del estudio identificar las características de los asociados de la Filial ASCUBI Villa Clara como usuarios potenciales para el diseño de una oferta de información Web. Diseñar una oferta de información Web para la filial ASCUBI Villa Clara a partir de la experiencia de los usuarios.

\section{Materiales y métodos}

La investigación se centra en un contexto investigativo particular: la Filial ASCUBI-Villa Clara. En esta filial la divulgación de información juega un rol fundamental debido a la cantidad y diversidad de instituciones de información con que cuenta la provincia. Las particularidades de cada una de las comunidades definidas se exponen a continuación:

- Profesionales y/o estudiantes de las especialidades de Técnico Medio en Bibliotecología, Técnico Medio en Gestión Documental; de la Enseñanza Técnico Profesional (ETP) o de la Licenciatura en Ciencias de la Información de la Enseñanza Superior en cualquier modalidad de estudio. Aquellos asociados a la filial aunque no cumplan los requisitos anteriores.

- Miembros de la Filial ASCUBI Villa Clara que ocupen cargos en la dirección. 
El estudio clasifica como investigación aplicada debido a que se propone una solución a un problema práctico. Se emplean métodos en el nivel teórico y empirico para la recogida de los datos, entre ellos: analítico-sintético, sistémico-estructural, histórico-lógico, inductivo deductivo, análisis documental clásico y la observación. Se aplican las técnicas de revisión de documentos, card sorting y la triangulación de información como vía para validar los resultados obtenidos. Se emplea la metodología de estudio de usuarios de Izquierdo-Alonso (1999) y la de Manso-Rodríguez (2008) para el diseño de la oferta de información.

\section{Resultados y discusión}

\section{Estudio de las necesidades de información de los asociados a la Filial ASCUBI Villa Clara.}

La Filial ASCUBI Villa Clara se crea el 24 de febrero de 1987 y fue una de las primeras en crearse en Cuba. Su creación consta en el registro de asociaciones del Ministerio de Justicia en la provincia. La filial fue una de las pocas con contar con un boletín oficial. El boletín ASCUBI Villa Clara es uno de los documentos en el ámbito provincial que intentó recoger algunos detalles históricos de la asociación.

En Aparicio-Martín (2012) se recogen múltiples datos estadísticos de la filial como la cantidad de asociados, premios obtenidos, directivos, entre otros. En la actualidad la membresía total de la filial es de 461 asociados. Las áreas de trabajo y sus miembros se pueden observar en la Tabla 1. 


\begin{tabular}{|l|l|}
\hline Área & Membresía \\
\hline Bibliotecas Públicas & 311 \\
\hline Centro de Información de Ciencias Médicas (CICM) & 36 \\
\hline Centro de Información Pedagógica & 15 \\
\hline $\begin{array}{l}\text { Universidad Central "Marta Abreu” de Las Villas } \\
\text { (UCLV) }\end{array}$ & 31 \\
\hline Empresarial y Científico & 16 \\
\hline Escolares & 39 \\
\hline Jubilados & 13 \\
\hline
\end{tabular}

Tabla 1: Asociados por áreas de trabajo (fuente: elaboración propia).

La filial villaclareña por varios años obtuvo reconocimientos a nivel nacional que avalan su labor. De forma sistemática fue la filial más destacada a nivel nacional. Una de las particularidades de la filial es que otorga un Premio Provincial, sobre este particular Bellas-Vilariño (2011) establece: "La filial villaclareña es una de las pocas que tiene un Premio Provincial para sus socios, el Premio Garófalo. Este premio se otorga a aquellos bibliotecarios que han contribuido al desarrollo de la bibliotecología en la provincia".

La filial se fortaleció con la creación en la UCLV de la especialidad de Licenciatura en Ciencias de la Información. Sus inicios datan de 2007 en el curso regular diurno (CRD). La especialidad se había creado en el curso para trabajadores (CPT) un año antes y con anterioridad en la modalidad de enseñanza a distancia.

La creación de esta carrera contribuyó a la superación profesional de los asociados. Constituyó un pilar para el desarrollo investigativo con la orientación teórica-metodológica de los profesores de la especialidad. Con la formación de pregrado se fortalecieron las relaciones entre las instituciones de información de la Región Central y la academia.

La profesión bibliotecaria en Villa Clara tiene varias personalidades que prestigian esta actividad profesional que aumenta el compromiso y sentido de pertenencia de los asociados con la filial provincial. Algunas de estas 
personalidades son: Manuel García Garófalo, Olinta Ariosa Morales y la recién desaparecida Juana Rosa Vázquez. Otros profesionales de importancia en el sector son Ramón Alberto Manso Rodríguez, Amed Abel Leyva y Cándida Judith Guerra Miranda, profesional aún activa dentro de la membresía de la asociación.

En el año 1990 se da a conocer el órgano de divulgación oficial de la filial con forma de boletín, titulado ASCUBI Villa Clara. El boletín fue el homólogo del boletín nacional ASCUBI Informa. Vale destacar que hasta donde se conoce esta fue la única filial con una publicación de tipo informativo para sus asociados. Su efímera existencia no demeritó la importancia que significó para la divulgación del acontecer de la esfera informativa.

Su formato fue de 160 X $208 \mathrm{~mm}$, en la primera edición, de 215 X $285 \mathrm{~mm}$ en la segunda. La paginación fluctuó entre las 20 y 25 páginas. La tipografía era de 10 puntos y esencialmente se emplearon caracteres en correspondencia con el teclado de máquinas de escribir utilizadas. El diseño de la cubierta se caracterizó por gran sencillez. Mostró una foto en blanco y negro del Conjunto Escultórico "Ernesto Ché Guevara". Este monumento es símbolo de Santa Clara y constituye un elemento identificativo del territorio.

Se aplica la metodología de Izquierdo-Alonso (1999) para la identificación de las necesidades de información de los asociados. Atendiendo al estudio de los autores se consultaron autores como Frías-Guzmán et al. (2015), PeraltaGonzález y Frías-Guzmán (2011), Peralta-González (2009), León (2008), Aparicio-Martín (2012), Paz-Enrique et al. (2014), Cárdenas-Pérez et al. (2011), Bellas-Vilariño (2006), Bellas-Vilariño (2007). Estas lecturas facilitaron la identificación de las temáticas más productivas en la Filial ASCUBI-Villa Clara, especialmente en los eventos bibliotecológicos que se celebran en la organización.

Los eventos principales donde participan miembros de la filial dentro de la provincia son: Taller de Conservación de Documentos, Encuentro Nacional de Conservación y Difusión del Patrimonio Documental y la Jornada Bibliotecológica Provincial. Este último evento es el más numeroso en cuanto a participantes y ponencias. Los autores Peralta-González y Frías-Guzmán (2011) identifican las instituciones más productoras. Sobre este particular refieren que "prevalece el protagonismo de las bibliotecas públicas. La UCLV y el CICM resaltan como las instituciones más productivas. Todavía es baja la 
producción científica de la provincia respecto del potencial institucional con que cuenta en esta área del conocimiento".

La creación a partir del 2010 de múltiples comisiones representó un crecimiento en la productividad de las jornadas. La creación de la especialidad de Licenciatura en Ciencias de la Información en la UCLV, favoreció la creación de una comisión para estudiantes.

Algunos medios de publicación consultados por los asociados a nivel provincial son El Directivo al Día (http://www.villaclara.cu/index.php/inicio) y la página del Archivo Histórico Provincial (http://archivohistorico.villaclara.cu/). En esta última se publican ponencias presentadas en el Encuentro Nacional de Conservación y Difusión del Patrimonio Documental.

Se localizaron autores o instituciones a nivel provincial con presencia en revistas o bases de datos de alto impacto. Los principales autores identificados son: Ramón Alberto Manso Rodríguez, Amed Abel Leiva Mederos, Grizly Meneses Placeres, María Josefa Peralta González, Maylín Frías Guzmán y Odalys Font Aranda. Las instituciones destacadas en cuanto a la producción científica son el Departamento de Ciencias de la Información y CDICT de la UCLV, la Biblioteca Provincial "Martí" y el Centro de Información de Ciencias Médicas. Las revistas donde mayor cantidad de publicaciones tienen los autores mencionados son:

- Revista ACIMED

- Revista Ciencias de la Información

- Revista Bibliotecas. Anales de Investigación

- Revista Ciencias Holguín

- Revista El profesional de la información.

- Revista Española de Documentación Cientifica

- Revista Interamericana de Bibliotecología

El análisis de los autores arrojó que los asociados de la Filial ASCUBI-Villa Clara, tienen una productividad científica que se refleja en la participación en eventos o en la publicación de artículos científicos.

Atendiendo al análisis de los documentos, se identificó la relación de ciertos aspectos de los mensajes científicos con las características psicocognitivas del receptor. A partir de la aplicación de una encuesta se identificaron dichas características. Del total de 461 asociados se encuestaron a 312, representando 
el $67.67 \%$ de la membresía total. En cuanto a sus edades físicas, los resultados pueden observarse en la Tabla 2.

\begin{tabular}{|l|c|c|}
\hline Edades & Cantidad & por ciento \\
\hline 18-24 años & 43 & 13,78 \\
\hline 25-35 años & 62 & 19,87 \\
\hline 36-45 años & 89 & 28,52 \\
\hline 46-55 años & 67 & 21,47 \\
\hline más de 55 años & 51 & 16,34 \\
\hline Total & 312 & 100 \\
\hline
\end{tabular}

Tabla 2: Edades de los asociados encuestados (fuente: elaboración propia).

Se observa un envejecimiento de los profesionales de la información en Villa Clara. El grupo de edades que menos cantidad de miembros tiene es el que comprende desde 18 a 24 años. Se delimita este grupo de edades porque constituyen nativos digitales (nacidos a partir de 1991) y son los que más habilidades tienen en el trabajo con los recursos electrónicos. La proporción entre nativos e inmigrantes digitales se observa en la Ilustración 1.

\section{Cantidad de asociados}

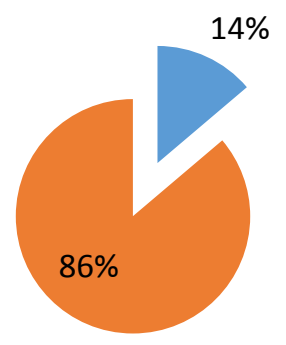

Nativos digitales

- Inmigrantes digitales

Ilustración 1: Proporción de nativos e inmigrantes digitales encuestados (fuente: elaboración propia). 
Se evidencia una alta desproporción entre ambos grupos. Para el diseño de una oferta de información deberá tenerse en cuenta este factor. La proporción de sexos de los encuestados puede observarse en la Tabla 3.

\begin{tabular}{|l|c|c|}
\hline Sexo & Cantidad de asociados & Por ciento \\
\hline Masculino & 36 & 11,53 \\
\hline Femenino & 276 & 89,5 \\
\hline Total & 312 & 100 \\
\hline
\end{tabular}

Tabla 3: Proporción de sexos de los encuestados (fuente: elaboración propia).

Existe un gran predominio de asociados del sexo femenino en las instituciones de información donde se aplicó la encuesta. La mayor cantidad de profesionales del sexo femenino se localizan en bibliotecas públicas.

La relación de áreas de trabajo y la proporción de los miembros encuestados se pueden observar en la Tabla 4.

\begin{tabular}{|l|c|}
\hline Área de trabajo & Cantidad de asociados \\
\hline Bibliotecas Públicas & 196 \\
\hline Ciencias Médicas & 32 \\
\hline Pedagógico & 12 \\
\hline UCLV & 24 \\
\hline Empresarial y científico & 11 \\
\hline Bibliotecas escolares & 27 \\
\hline Jubilados & 10 \\
\hline Total & 312 \\
\hline
\end{tabular}

Tabla 4: Encuestados por áreas de trabajo (fuente: elaboración propia).

De las áreas de trabajo son las bibliotecas públicas donde mayor cantidad de asociados se encuestan. Muchas de las encuestas realizadas fuera del territorio del municipio de Santa Clara fueron realizadas vía correo electrónico. Esto supone una limitación en cuanto al contacto cara a cara y a la explicación del 
contenido de las encuestas. Se redujo, por tanto, la explicación y entendimiento de las orientaciones enviadas por esta vía. Del área bibliotecas públicas se encuestaron asociados de la Biblioteca Provincial "Martí" de Villa Clara, Remedios, Corralillo, Sagua la Grande, Ranchuelo, Santo Domingo, Manicaragua, Caibarién, Cifuentes, Encrucijada y la Sala de Lectura del Condado.

En cuanto a las bibliotecas universitarias se encuestaron asociados del Centro de Información y Documentación Científico Tecnológica (CDICT) de la UCLV, Centro de Información Pedagógica (CDIP) de la Universidad de Ciencias Pedagógicas (UCP) y el CICM. Del área UCLV se encuestaron además varios estudiantes de Ciencias de la Información. Esto se efectuó por el papel protagónico que tendrán como futuros miembros de la Filial.

De las instituciones museables se encuestaron asociados del Museo de las Parrandas de Remedios, Museo Municipal de Remedios, Museo Provincial de la Música de Remedios, Museo Histórico Provincial "Abel Santamaría" en Santa Clara. Se entrevistaron asociados de las instituciones archivísticas con que cuenta la provincia: Archivo Histórico de Remedios, Archivo Histórico Provincial. Del área de trabajo empresarial y científico se entrevistaron asociados del Departamento de Ciencias de la Información de la UCLV, del CIGET de Santa Clara y del Sectorial Provincial de Cultura.

En cuanto al nivel profesional de los encuestados se identificó el predominio de licenciados, aunque muchos no son graduados de la especialidad. Aun así se desempeñan en instituciones de información con diversos roles. La proporción del nivel profesional y la cantidad de encuestados se muestra en la Ilustración 2. 


\section{Nivel cultural de los encuestados}

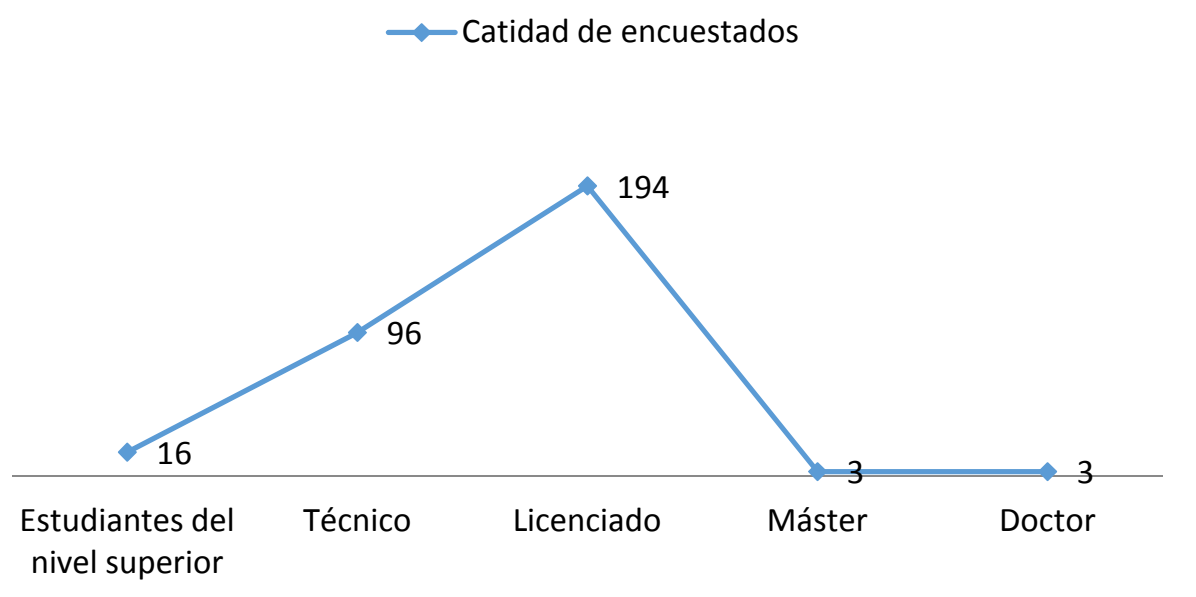

Ilustración 2: Nivel cultural de los encuestados (fuente: elaboración propia).

La gran cantidad de especialistas favorece la implementación de una oferta que refleje comunicaciones científicas. La superación de los miembros es uno de los objetivos de la filial. La creación de un canal de comunicación científica impulsará la productividad de los asociados. En la encuesta aplicada, se les pidió a los 312 encuestados que establecieran un orden de prioridad (1 máximo al 10 mínimo) sobre su experiencia en cuanto a:

A. Navegar en Internet.

B. Acceder a información digital.

C. Buscar y recuperar información en ambiente Web.

D. Actualizar las herramientas tecnológicas de las que disponen.

A partir de los resultados se pueden clasificar las respuestas de la siguiente forma:

- De 1 hasta 3: se clasifica de bajo

- De 4 hasta 6: se clasifica de medio

- De 7 hasta 10: se clasifica de alto

Aunque varios de los encuestados dejaron opciones en blanco, la opción que obtuvo las respuestas más satisfactorias fue la $\mathrm{A}$ referida a la experiencia a navegar en internet. La respuesta menos favorecida fue la $\mathrm{D}$ referida a la actualización con las herramientas tecnológicas. En la opción A solo 7 personas se clasificaron con experiencia baja, en la B: 11, en la C: 12 y en la D: 67, siendo este el resultado más bajo. Los resultados obtenidos pueden ser visualizados en la Ilustración 3. 


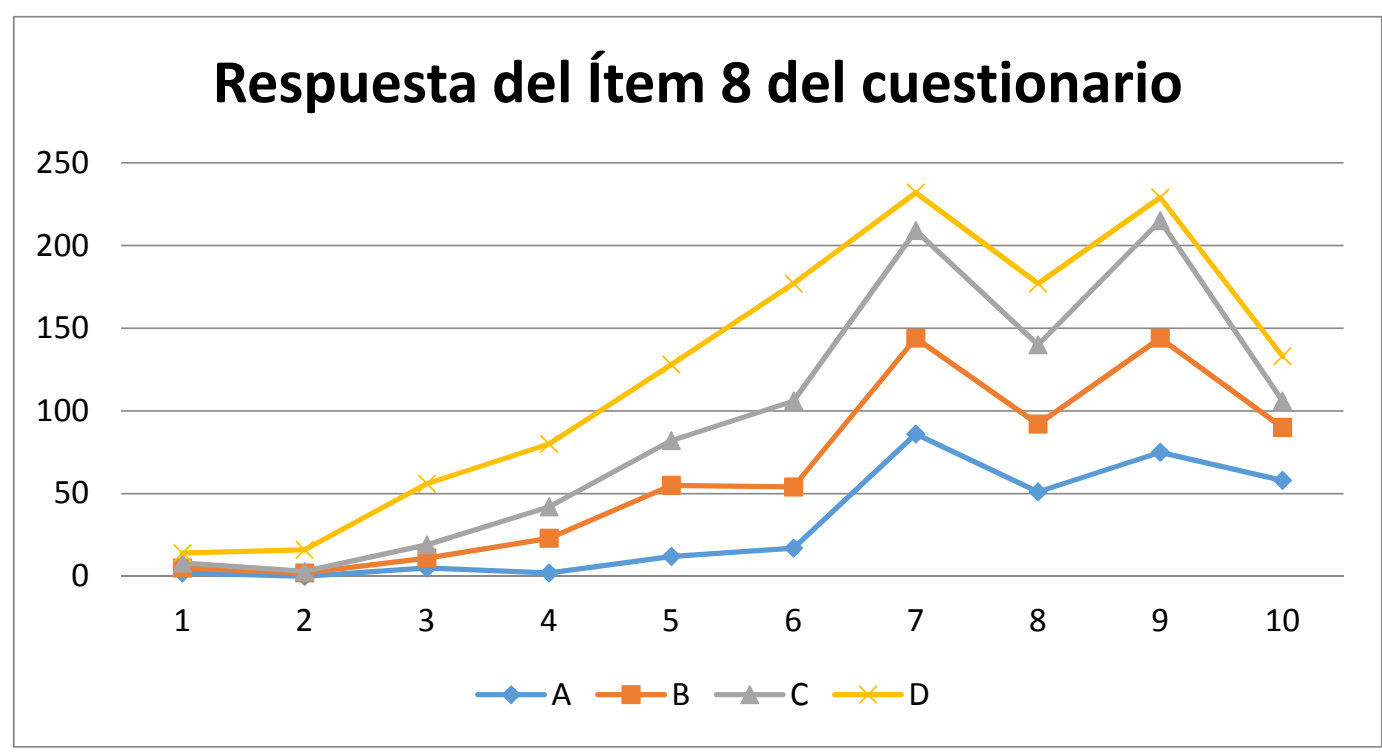

Ilustración 3: Resultados obtenidos de las respuestas del Ítem 8 del cuestionario aplicado a los asociados de la Filial ASCUBI-Villa Clara (fuente: elaboración propia).

En sentido general las respuestas pueden clasificarse de Media a Alta. Hasta el valor 7 se detectó un crecimiento. Luego de este valor la tendencia fue a disminuir. La moda está constituida por el valor 7 y la mayoría de las respuestas están en el rango de Alto.

Los resultados obtenidos favorecen la propuesta de creación de un boletín científico en formato electrónico e impreso para la Filial ASCUBI Villa Clara. Se identificaron 3 boletines creados para la filial con una existencia efímera: el boletín ASCUBI Villa Clara, el Exlibris y el Boletín Informativo.

Se plantearon categorías de usuarios potenciales, definiéndose la siguiente segmentación:

Atendiendo al desempeño profesional:

- Profesionales del Sistema Provincial de Bibliotecas Públicas en Villa Clara.

- Profesionales que se desempeñen en los museo, archivos y centros de información y documentación

- Trabajadores del nivel técnico-profesional que se desempeñen en instituciones de información de la provincia.

- Profesionales del sector de la Cultura.

- Investigadores y trabajadores de la información.

- Estudiantes del nivel superior de la especialidad de Ciencias de la Información. 
- Estudiantes del nivel técnico-profesional de las especialidades de Bibliotecología y Gestión Documental.

Atendiendo a las edades se definieron dos categorías de usuarios: nativos e inmigrantes digitales.

Atendiendo al nivel profesional de los usuarios se plantean las siguientes categorías:

- Licenciados en Ciencias de la Información.

- Másteres en Ciencias de la Información.

- Doctores en Ciencias de la Información.

- Trabajadores del nivel técnico-profesional de las especialidades de Bibliotecología y Gestión Documental

- Estudiantes del nivel técnico-profesional de las especialidades de Bibliotecología y Gestión Documental y del nivel superior de la Licenciatura en Ciencias de la Información.

- Otros (profesionales de otras áreas del conocimiento que se relacionen con la labor del profesional de la información).

En cuanto al área geográfica se plantearon las siguientes categorías:

- Profesionales que se desempeñen en los museos, archivos, bibliotecas y centros de información y documentación en Santa Clara.

- Profesionales que se desempeñen en los museos, archivos, bibliotecas y centros de información y documentación en las delegaciones municipales de base.

- Profesionales del sector de la Cultura en Santa Clara.

- Profesionales del sector de la Cultura en los municipios.

- Estudiantes del nivel superior de la especialidad de Ciencias de la Información en las provincias Cienfuegos, Sancti Spíritus y Villa Clara. Las principales publicaciones a nivel nacional, que consultan y donde por lo general publican los profesionales de la información son:

- Revista ACIMED

- Revista Ciencias de la Información

- Revista Bibliotecas. Anales de Investigación

- Revista Ciencias Holguín

- Revista Librínsula

A nivel internacional las publicaciones más consultadas según el criterio de los usuarios son: 
- Revista El profesional de la información.

- Revista Española de Documentación Científica

- Revista Interamericana de Bibliotecología

- Journal of Documentation

- Revista Biblios

- Investigación Bibliotecológica

- Revista e-Ciencias de la Información

La clasificación general de los tipos de usuarios resultante es la siguiente:

- Usuarios internos: personal que labora en el funcionamiento del boletín (editores, correctores, revisores, árbitros, etc.). Estos usuarios también pueden proponer contribuciones para la oferta.

- Usuarios externos: colaboradores, autores y lectores para el boletín.

A partir de las consideraciones anteriores y en consonancia con las necesidades de información de los usuarios se propone la creación de una oferta de información Web. La propuesta deberá ser un boletín científico electrónico que facilite a los usuarios la publicación de sus investigaciones. Previo al diseño de la oferta se identificaron los requerimientos y preferencias de los usuarios, mediante la encuesta. Los requerimientos se recuperaron mediante la pregunta 9: ¿Cómo desea tener acceso al Boletín? Los resultados se pueden observar en la Tabla 5 .

\begin{tabular}{|l|c|}
\hline Opciones & Frecuencia \\
\hline Correo electrónico & 289 \\
\hline $\begin{array}{l}\text { En formato electrónico mediante un sitio } \\
\text { Web }\end{array}$ & 274 \\
\hline Impreso & 306 \\
\hline Otro & 6 \\
\hline
\end{tabular}

Tabla 5: Resultados de la pregunta 9 referida al acceso al boletín por parte de los asociados (fuente: elaboración propia).

El formato impreso fue el más favorecido con un registro de 306, representando el $98.7 \%$ de los encuestados, esto se relaciona con el acceso que presentan los asociados de las delegaciones municipales de base. La opción 
Otro fue la menos favorecida, en las encuestas realizadas cara a cara, los encuestados manifestaron que desconocen en qué otro formato pueden consultar el boletín. Solo 2 usuarios propusieron otro formato: publicado en un CD.

De acuerdo con los datos que posee el Buró Ejecutivo Provincial de la filial, se presentan las potencialidades tecnológicas de la filial. Los usuarios potenciales ascienden a la cifra de 125 y pueden consultar la oferta en línea, y 336 usuarios la pueden recibir vía correo electrónico. A pesar de ello, la situación tecnológica de las delegaciones de base localizadas en los municipios no es muy favorable. Instituciones de información como museos y archivos no poseen conectividad.

A partir de los resultados se estima enviar el boletín publicado o una síntesis del mismo mediante el correo electrónico. Se dispondrá una plataforma Web para alojar el boletín y se realizará un número impreso editado de forma anual. Esto último para favorecer el acceso de aquellos que lo solicitaron mediante esta vía. Por razones económicas de la filial no se puede imprimir más de una vez al año.

De acuerdo con las preferencias, se les preguntó a los encuestados el tipo de información que debía tener la oferta. Los resultados en este ítem pueden observarse en la Tabla 6.

\begin{tabular}{|l|c|}
\hline Tipo de información & Frecuencia \\
\hline Noticias de la filial & 301 \\
\hline Artículos de investigación & 264 \\
\hline Noticias nacionales del sector & 239 \\
\hline Nota editorial & 228 \\
\hline Biografías a personalidades destacadas & 214 \\
\hline Noticias internacionales del sector & 213 \\
\hline Entrevistas a personalidades destacadas & 205 \\
\hline Promociones de servicios, eventos y productos & 198 \\
\hline
\end{tabular}


Tabla 6: Resultados de la pregunta referida al tipo de información que prefieren los usuarios que aparezcan (fuente: elaboración propia).

Noticias (de la filial) fue el tipo de información que más solicitaron y en menor medida la Nota editorial. El formato de boletín se compondrá por secciones que cumplan con las expectativas de los usuarios, estableciéndose secciones a partir de los resultados de este ítem. Se les preguntó a los usuarios si deseaban que el boletín se descargue en formato PDF, para lo que la totalidad respondió afirmativamente. Se preguntó el rol que desearían ejercer dentro del boletín, como muestra la Tabla 7.

\begin{tabular}{|l|c|}
\hline Rol & Frecuencia \\
\hline Lector & 303 \\
\hline Autor & 181 \\
\hline Revisor & 23 \\
\hline Árbitro & 16 \\
\hline Editor & 11 \\
\hline Editor de una sección & 6 \\
\hline $\begin{array}{l}\text { Responsable de una } \\
\text { sección }\end{array}$ & 9 \\
\hline Revisor de una sección & 4 \\
\hline
\end{tabular}

Tabla 7: Resultados de la pregunta 12 referida al rol que desearían ejercer los encuestados en el boletín (Fuente: elaboración propia).

El hecho de que el rol Lector sea el más favorecido es debido a que varios encuestados marcaron más de una opción. Casi la totalidad desea ser lector del boletín. En menor medida los usuarios desean ser revisores de una sección de la publicación.

Sobre el formato de la visualización del boletín, un total de 298 encuestados estima que debe poseer texto e imágenes. La última pregunta de la encuesta se 
refiere a las temáticas que debe tratar el boletín. Las respuestas más recurrentes fueron las siguientes:

- Alfabetización informacional

- Tecnologías de la información y las comunicaciones

- Conservación de documentos

- Historia

- Restauración de documentos

- Procesamiento de la información

- Estudios métricos

- Promoción de lectura

- Aspectos teóricos de la información

- Arquitectura de información

- Comportamiento humano informacional

- Desarrollo de colecciones

- Procesos archivísticos

Luego de identificar las necesidades de información de los usuarios se procede al diseño del boletín como oferta de información que satisfaga sus demandas.

\section{Diseño de la oferta de información.}

Para el diseño de la oferta de información se emplea la metodología planteada por Manso (2008). La misma cuenta con 6 pasos que se desarrollan a continuación.

Atendiendo a la fase de documentación se realiza un estudio de usuarios donde se identifican las principales necesidades de información de los miembros de la Filial ASCUBI Villa Clara. Se identifican los recursos necesarios para la creación de la oferta (humanos, financieros, tecnológicos). Los recursos humanos necesarios para la implementación de la oferta que se propone están relacionados con las categorías de lectores, autores e investigadores, personal técnico encargado de mantenimiento de la oferta y profesionales encargados del proceso editorial

Los lectores son los usuarios que consultan el contenido del boletín, pero no están involucrados en los procesos editoriales del mismo. Los autores e 
investigadores son los miembros de la filial que contribuirán con el boletín proponiendo artículos, entrevistas, etc. Los lectores, autores e investigadores tendrán libre acceso al contenido de las informaciones del boletín, pero no podrán modificar ni cambiar aspectos de forma o contenido en este.

El personal técnico encargado del mantenimiento del boletín puede ser una o varias personas que actualicen el boletín y publiquen los números en la plataforma tecnológica donde se aloje. Los profesionales que intervengan en esta oferta serán definidos bajo la categoría de soporte técnico. Deberán tener conocimientos amplios sobre el Open Journal Systems (OJS) y su funcionamiento. Se escoge el OJS como herramienta atendiendo a la experiencia que tienen los usuarios, la mayor parte de las revistas y publicaciones que más utilizan emplean el OJS.

Para llevar a cabo el proceso editorial se establecen los roles de editor en jefe, correctores, editores, revisores, árbitros y responsable de secciones. El editor en jefe es el responsable principal de la oferta. A este rol se subordinará el personal de soporte técnico que deberá atender a las sugerencias realizadas por el editor. Los roles de correctores, editores, revisores, árbitros y responsable de secciones; generalmente se decidirán por el comité editorial. Los roles antes mencionados pueden proponerse a título personal en el momento de inscripción en el boletín. El OJS brinda la posibilidad para que los profesionales que así lo estimen, soliciten ser lectores, autores o revisores del boletín.

La oferta que se propone no requerirá de altos gastos económicos. El personal propuesto para el boletín realizará esta actividad de forma desinteresada, como parte de sus intereses investigativos. La oferta no contempla el pago por las contribuciones hechas por los autores. El boletín se sustenta en el acceso abierto de todo el material en el momento que sea publicado.

Los principales gastos del boletín estarán en la solicitud de servicios de diseño gráfico para su edición impresa. La impresión del boletín también generará gastos de papel, tinta y los servicios propios de impresión. La Filial ASCUBI Villa Clara deberá sufragar estos gastos. Se propone para ello que la filial asigne un fondo destinado a la edición impresa del boletín. Se propone, para minimizar gastos, la impresión de un volumen anual con todos los números publicados en ese período. Los ejemplares deberán cubrir- al menos- uno para cada delegación de base.

Los recursos tecnológicos necesarios para la implementación del boletín son: 
- Hosting para la ubicación del boletín.

- Servidor donde alojar la dirección del boletín.

- Salida internacional.

- Conexión nacional para los miembros de las delegaciones de base.

Atendiendo al diseño de la oferta se realizó una política informacional para el boletín arrojando como resultados los documentos de Descripción de la oferta de información, Proceso de Evaluación por pares, Política de Acceso abierto, Políticas de sección, Normas de redacción para el envío de contribuciones, Directrices para la revisión de las contribuciones.

Descripción: Reportes de Información es un boletín científico, de acceso abierto y sin fines de lucro para los profesionales de la información. Pertenece a la Asociación Cubana de Bibliotecarios (ASCUBI), filial Villa Clara. Es asesorado por el Departamento de Ciencias de la Información de la Universidad Central "Marta Abreu" de Las Villas (UCLV). El boletín se publica en formato electrónico e impreso. Cuenta con las secciones: EDITORIAL, ARTÍCULOS CIENTÍFICOS, ARTÍCULOS DE REVISIÓN, ARCHIVO CIENTÍFICO, NOTICIAS, PERSONALIDADES, ENTREVISTAS y PROMOCIONES.

La publicación es el órgano oficial de divulgación de la Filial ASCUBI Villa Clara. Reportes de Información es la continuación del boletín impreso ASCUBI Villa Clara, que fungió como órgano de los profesionales de la información en la provincia en la década del 90 del siglo XX. La propuesta actual pretende rescatar este boletín.

La publicación del boletín se basa en la herramienta OJS versión 2.4.5.0, que es un gestor de publicaciones seriadas de acceso abierto y un software desarrollado, financiado y distribuido de forma gratuita por el proyecto Public Knowledge Project.

Las contribuciones y recursos de información que generará el boletín serán infografías, notas editoriales, artículos de investigación, artículos de revisión, noticias, entrevistas, reseñas, biografías, promociones de eventos, así como promoción de fuentes y servicios de información. La política fundamental de la oferta es la construcción colectiva, para ello el comité editorial lanza la convocatoria a sus afiliados (usuarios potenciales) a colaborar con el boletín. Cada contribución deberá ser revisada y asesorada, garantizando un mínimo de calidad y pertinencia con los objetivos del boletín. Se recogerán los criterios 
de los usuarios para la evaluación y rediseño, en caso de ser necesario, del boletín. Delimitación del alcance y objetivos de la oferta.

Misión: satisfacer las demandas de publicación y consulta de los profesionales de la información de la Filial ASCUBI Villa Clara. Propiciar el intercambio de experiencias entre los profesionales de la información de la provincia, a partir del desarrollo de un canal de comunicación.

Visión: el boletín es una publicación seriada de referencia en América Latina para los estudiantes y profesionales de la información, está posicionada en importantes bases de datos internacionales que le aportan alta visibilidad e impacto a nivel internacional. Está traducido al inglés y portugués.

Objetivos:

- Propiciar el intercambio de experiencias en lo relacionado con las Ciencias de la Información.

- Establecer un canal de comunicación científica para los asociados de la filial provincial.

- Favorecer la productividad científica de los profesionales de la información de la Filial ASCUBI Villa Clara.

- Incentivar la productividad científica de los profesionales de la información de la Filial ASCUBI Villa Clara.

- Promocionar eventos, servicios y productos de información para los asociados de la Filial ASCUBI Villa Clara.

- Promocionar noticias y actividades del sector relacionadas con los asociados de la Filial ASCUBI Villa Clara.

Se definieron las responsabilidades correspondientes al capital humano que laborará en el boletín. En el rol de soporte técnico es establecido como responsable a un estudiante de 5to año de Ciencias de la Información de la UCLV como parte de su trabajo de diploma. Posteriormente el Departamento de Ciencias de la Información deberá asignar otra persona. Se definió el comité editorial del boletín.

Temáticas de publicación: Reportes de Información acepta contribuciones sobre Bibliotecología, Archivística, Museología y Ciencia de la Información. Además de otros temas relativos a la información.

Periodicidad: cuatrimestral (febrero, junio y octubre).

Arbitraje: Es una publicación arbitrada que utiliza el sistema de doble ciego, conforme a las normas de publicación de la Norma ISO 690. 
Proceso de Evaluación por pares: las contribuciones son revisadas a través del doble ciego donde intervienen dos revisores por cada contribución. En caso de que los revisores discrepen en criterios, se solicita la opinión de un árbitro para la decisión final. Los autores serán informados de la decisión en un plazo no mayor de 6 semanas a partir de la recepción de la contribución. Actualmente cuenta con un total de 9 revisores y 6 árbitros de alto prestigio académico.

Política de Acceso abierto: este boletín proporciona acceso abierto inmediato a su contenido, basado en el principio de ofrecer al público un acceso libre a las investigaciones para ayuda a un mayor intercambio global de conocimiento.

Otros documentos resultantes de la fase de diseño son la política de sección y las normas de redacción.

Se elaboró un informe en el que se disponen las acciones y sus responsables. Para cada actividad se dispone una fecha de cumplimiento donde se chequeará periódicamente el estado de desarrollo de la acción. Se definieron roles por actividad profesional y se entregaron las funciones a los profesionales involucrados. Las funciones propuestas para los especialistas son:

- Profesional de la información: gestor del boletín, rol de dirección.

- Profesional de Ciencias de la Computación: solicitud de una dirección electrónica. Instalación del OJS y creación de la base de datos. Otorgamiento de permisos de administración al profesional de la información para la implementación del boletín. Asesoramiento profesional sobre el uso del OJS y alojamiento Web del boletín.

- Diseñador gráfico: realizar la identidad visual del boletín. Para ello definir un logo y elaborar productos comunicativos como slides o banners para que sean ubicados en el boletín electrónico.

- Comunicador social: realizar una campaña de promoción del boletín en correspondencia con el trabajo realizado por el diseñador gráfico.

Se diseñó la identidad visual de la oferta, estableciendo el logo y varios productos comunicativos. La identidad visual de la oferta puede observarse en la ilustración 4. 


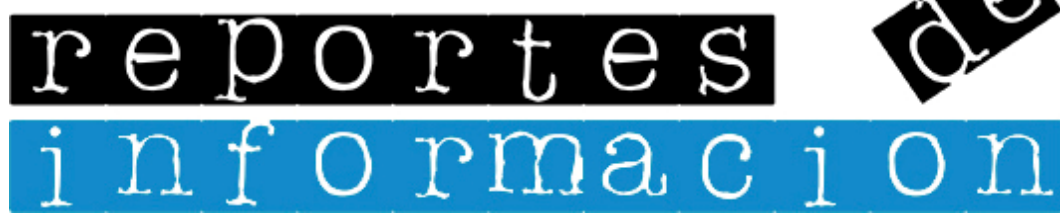

Ilustración 4: Identificador de la oferta (fuente: elaboración propia).

Se realiza la AI del boletín basada en la estructura del OJS. Se realizaron pruebas de control y test de usuarios con la finalidad de verificar el correcto mantenimiento del boletín. Ya implementado el boletín, debe establecerse un período de prueba de seis meses de duración. En este período debe realizarse un seguimiento del boletín a partir de su funcionamiento.

El entrenamiento que deberá recibir el personal es mínimo, téngase en cuenta que la oferta que se diseña se basa en la experiencia de los usuarios. Se establecen y diseñan aspectos en los que la mayoría de los usuarios están familiarizados, esto garantiza la usabilidad de la oferta que se diseña. Se identifican los usuarios que necesitan recibir conocimientos previos para el uso de la oferta y se imparten cursos de superación sobre OJS y comunicación científica.

La promoción del boletín deberá iniciar antes que sea implementado. Se indicó una breve reseña del servicio y se brindaron contactos para atender las inquietudes de los usuarios. Luego de haberse implementado el boletín, se informó a los usuarios potenciales de su existencia y finalidad. Se utilizaron para ello todos los canales de información viables en el contexto. El especialista de Comunicación Social fue importante en la promoción del boletín. De igual forma se recomienda que profesionales de estudios socioculturales apoyen esta actividad para futuras acciones promocionales. Los canales de información que deben priorizarse para la promoción del boletín son los siguientes:

- Publicaciones seriadas similares.

- Publicación de un artículo en la sección de Experiencias para Divulgar de la Revista Biblioteca. Anales de Investigación.

- Grupo en Facebook de la Filial ASCUBI Villa Clara.

- Canales de comunicación del Ejecutivo Provincial de la ASCUBI con las delegaciones municipales de base.

- Presentación de ponencias en eventos científicos de la especialidad.

- Lista de correos para los asociados de la filial. 
Debido a las dificultades tecnológicas de los asociados de la filial se plantean varias alternativas para facilitar la consulta del boletín:

- Divulgación en las delegaciones de base de las normas de publicación y formas de realizar contribuciones a través de los canales de información establecidos para los miembros de la filial.

- Divulgación en eventos y encuentros provinciales del boletín.

- Entrega de las normas y la caracterización del boletín en todas las delegaciones de base.

- Edición electrónica de los números publicados en el boletín, distribuido por correo electrónico o dispositivos de almacenamiento digital.

- Edición impresa (anual) del boletín.

La evaluación de la oferta se centró en 3 aspectos fundamentales: diseño gráfico, AI y funcionamiento. La evaluación del diseño gráfico se realizó a través del método criterio de especialistas, definiéndose para esto tres comunidades epistémicas:

- Profesores e investigadores del Departamento de Ciencias de la Información de la UCLV

- Ejecutivo provincial de la ASCUBI

- Diseñadores gráficos y comunicadores sociales

Se envió la propuesta de identificador vía correo electrónico a los profesionales relacionados con estas comunidades. Del Departamento de Ciencias de la Información se seleccionaron 5 profesionales con mayor experiencia laboral, del Buró Provincial de la ASCUBI se envió al Presidente de la Filial, a la Responsable de Superación y al encargado de Divulgación. En cuanto la otra comunidad se envió a 2 licenciados en Comunicación Social, 1 egresado del Instituto Superior de Diseño y a 1 Doctor en Ciencias de la Educación que ha tenido experiencia profesional en el diseño Web.

A partir de los criterios recogidos se rediseñó la propuesta de identificador visual. La mayoría de los especialistas concuerdan en que la idea está bien desarrollada, los elementos visuales bien distribuidos, el tipo de letra bien empleado, la idea es clara y accesible a los usuarios o público y posee cualidades y calidad en cuanto al diseño. Se diseñó además una convocatoria y un plegable para la divulgación y el recibo de contribuciones para el primer número del boletín. 
Atendiendo a la AI y el funcionamiento de las ofertas, se realizó una investigación con las propuestas de identificador visual y AI. Se les envió por correo electrónico a profesores del Departamento de Ciencias de la Información, a los directivos de la filial y a especialistas AI y programación Web. Los criterios recogidos se referían fundamentalmente a:

- Colores utilizados en la identidad visual del sitio.

- Tipología de plantilla empleada en el OJS.

- Contenido de las normas para los autores.

- Información sobre el consejo editorial.

- Etiquetas del sitio.

- Tipografía empleada.

Los resultados de la técnica de card sorting estuvieron enfocados hacia el cambio de etiquetas textuales, así como sugerencias de estructuración y eliminación de ambigüedades en las interfaces. Luego de atender a las sugerencias, se les solicitó que accedieran al boletín y valoraran la nueva propuesta. Esto arrojó que existe una homogeneidad en la conformidad de los entrevistados con todas las páginas del boletín. Algunas de las interfaces de la oferta se observan en la Ilustración 5. 


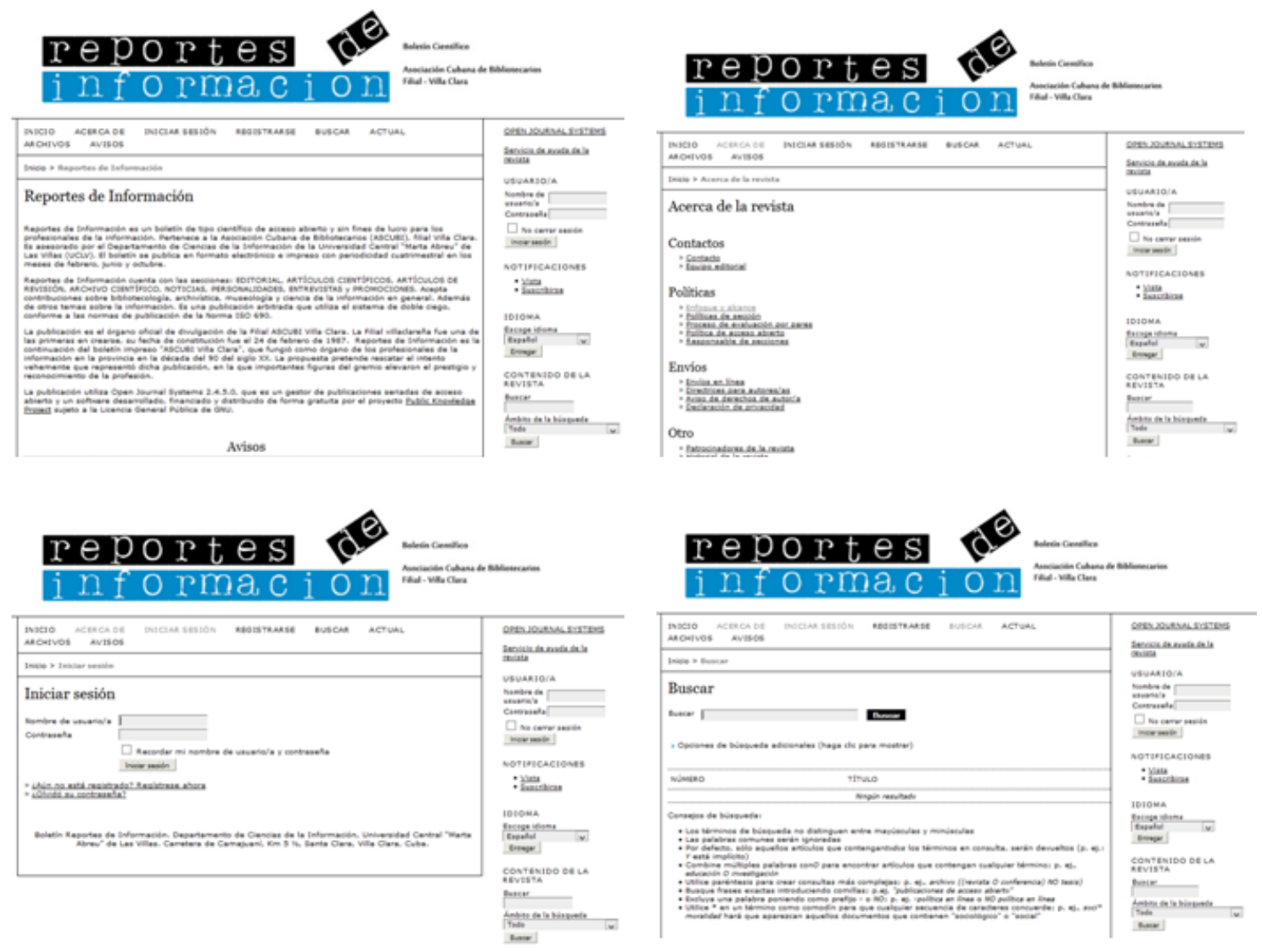

Ilustración 5: Interfaces de la oferta (fuente: elaboración propia).

Para combinar el card sorting con la observación directa se presentó el boletín en la XV Jornada Bibliotecológica de la Provincia de Villa Clara. Se analizaron los indicadores: tiempo de respuesta, dudas, aceptación visual y expresada. Se observó que los participantes manifestaron aceptación con el boletín propuesto. En este tipo de card sorting los usuarios abiertamente tuvieron la posibilidad de emitir opiniones libremente. Para la evaluación final del funcionamiento se solicita a un grupo de usuarios que interactúen con la oferta diseñada, pudiendo ser constatado que la oferta se encuentra al $100 \%$ de su funcionamiento. Se establece un período de prueba de seis meses donde la oferta solo estará visible desde la UCLV para poder continuar perfeccionándola y luego hacerla disponible desde internet.

\section{Conclusiones}

El diseño de ofertas de información basado en la experiencia de los usuarios y centrado en sus necesidades, favorece la usabilidad, la participación y minimizan los recursos que se destinan al entrenamiento del personal. 
Los miembros de la Filial ASCUBI Villa Clara carecen de un boletín oficial para divulgar aspectos de la organización y potenciar la investigación científica. Los miembros de la organización cuentan con una alta experiencia en el uso del OJS y que contempla las características y necesidades de los usuarios.

Se diseña la oferta: Reportes de Información, basada en la experiencia de los usuarios. La oferta diseñada favorece el intercambio de los usuarios, la elaboración colectiva de conocimientos y la divulgación de información de la filial.

\section{Bibliografía}

APARICIO-MARTÍN, L. A., 2012. La Asociación Cubana de Bibliotecarios (ASCUBI) en Villa Clara (1987-2007). Trabajo de diploma presentado en adopción al título de Licenciado en Ciencias de la Información. Departamento de Ciencias de la Información. Universidad Central "Marta Abreu" de Las Villas, Cuba.

BELIMPASAKIS, P. Y SAARANEN, A., 2010. Sharing with people: a system for user-centric content sharing. Multimedia Systems, 16(6), 399-421.

BELLAS-VILARIÑO, M., 2006. La Asociación Cubana de Bibliotecarios. Bibliotecas. Anales de Investigación, 2(2006), 1-16.

BELLAS-VILARIÑO, M., 2007. La Asociación Cubana de Bibliotecarios, un enfoque desde la teoría de las organizaciones. Bibliotecas. Anales de Investigación, 3(2007), 1-20.

BELLAS-VILARIÑO, M., 2011. La Asociación Cubana de Bibliotecarios: examen histórico de su papel en el desarrollo del campo bibliotecológico nacional. Trabajo de diploma presentado en adopción al titulo de Máster en Bibliotecología y Ciencia de la Información. Departamento de Bibliotecología y Ciencias de la Información. Universidad de La Habana, Cuba.

CABRERA, F.A., DONOSO, T., ANEAS, A. y DEL CAMPO, J., 2010. Valoración de la satisfacción de usuarios de programas sociales: propuesta de un modelo de análisis. Revista de Educación, 351(2010), 311-336. 
CALVA-GONZÁLEZ, J.J., 2013. Estudios de usuarios en diferentes comunidades: necesidades de información y comportamiento informativo. México: Instituto de Investigaciones Bibliotecológicas y de la Información.

CÁRDENAS-PÉREZ, M.R., ESTÉVEZ-CHAVIANO, V. y HERNÁNDEZRODRÍGUEZ, M., 2011. Boletín Informativo: una herramienta indispensable de comunicación de la Biblioteca Provincial Martí. En: M ARTILES. XI Jornada Científico-Bibliotecológica Provincial. Santa Clara: Editorial Feijóo. 14p.

REY-MARTÍN, C., 2000. La satisfacción del usuario: un concepto en alza. Anales de Documentación, 3(2000), 139-153.

FIGUEREDO, A.L., FIGUEREDO, M.C. y APONTE, C., 2013. La biblioteca universitaria como institución de apoyo a la docencia y la investigación. Revista Bibliotecas. Anales de investigación, 31(1), 1-8.

FRÍAS-GUZMÁN， M., ALOMÁ-CIRES， C., TAMAYO-RUEDA， D., APARICIO-MARTÍN, L. A., PAZ-ENRIQUE, L. E., 2015. Apuntes históricos sobre el boletín ASCUBI Villa Clara. En: M ARTILES. Forum de Historia de la UCLV. Santa Clara: Editorial Feijó. 18p.

GALLO, J.P., 2008. Innovación en los servicios de bibliotecas públicas orientadas al usuario. El caso de la Biblioteca Regional de Murcia. El profesional de la información, 27(2), 144-154.

GONZÁLEZ, A., 2011. La perspectiva del usuario y del sistema en la investigación sobre el comportamiento informacional. Teoría de la Educación. Educación y Cultura en la Sociedad de la Información, 12(1), 9-27.

HARSH-BARDHAN, A., y MISHRA, J. K., 2012. Oh! Web 2.0, Virtual Reference Service 2.0, Tools \& Techniques (II). Journal of Library \& Information Services in Distance Learning, 6(1), 149-171.

IZQUIERDO-ALONSO, M., 1999. Una aproximación interdisciplinar al estudio del usuario de información: bases conceptuales y metodológicas. Investigación Bibliotecológica, 13(26), 112-134.

LEÓN, M., 2008. Comportamiento de las investigaciones científicas y otros estudios en el Sistema de bibliotecas públicas de Cuba: 1990-2005. Bibliotecas. Anales de Investigación, 4(2008), 17-27.

MANSO-RODRÍGUEZ, R.A., 2008. Referencia Virtual: un enfoque desde las dimensiones asociadas a la gestión de información. Ciencias de la Información, 39(2), $59-68$. 
MANSO-RODRÍGUEZ, R.A., 2012. Bibliotecas, fomento de la lectura y redes sociales: convirtamos amigos en lectores. El profesional de la información, 21(4), 401405.

PAZ, L.E., HERNÁNDEZ, E.A. y MANSO, R.A., 2014. Diseño de la Arquitectura de Información para el Producto: InfoFEU-UCLV. Revista Infociencia, 1(19), 1-12.

PERALTA, M.J.; SOLÍS, F.M. y PERALTA, L.M., 2011. Visibilidad e impacto de la producción científica de la Universidad Central "Marta Abreu" de Las Villas durante el período 2000-2008. Acimed, 1(22), 60-78.

PERALTA-GONZÁLEZ, M. F., y FRÍAS-GUZMÁN, M., 2011. Acercamiento bibliométrico a la investigación científica en las Jornadas Científico-Bibliotecológicas Villaclareñas. Bibliotecas. Anales de Investigación, 7(7), 71-78.

RENDÓN, M.A. y HERNÁNDEZ, P., 2010. Sense-making: ¿metateoría, metodología o heurística? Revista Investigación Bibliotecológica, 24(52), 61-81.

WANG, X. y KLINC, R., 2012. Special issue: eLearning 2.0: Web 2.0-based social learning in built environment. Journal of Information Technology in Construction, 17(2012), 386-387. 